\title{
PERFIL DE CONGELAMENTO DO CARRÉ SUÍNO: UM ESTUDO DE CASO
}

\author{
RENATO CARLESSO* \\ WEBER DA SILVA ROBAZZA** \\ ALESSANDRO CAZONATTO GALVÃO***
}

\begin{abstract}
Este estudo teve como objetivo verificar as características de um túnel de congelamento de uma empresa da região oeste do estado de Santa Catarina, Brasil, por meio da realização de análise do perfil da velocidade de congelamento do carré suíno e avaliar a influência da velocidade do ar no congelamento em diferentes pontos do túnel. A análise detalhada dos dados permitiu observar a existência de dois períodos distintos de congelamento para todos os pontos estudados. Os resultados indicam que o congelamento do carré suíno no túnel estudado é inadequado nos três pontos investigados. Uma análise da velocidade média do ar indica que o túnel estudado opera de forma bastante heterogênea, proporcionando um efeito direto na qualidade e no tempo de congelamento.
\end{abstract}

* Engenheiro de alimentos, Departamento de Engenharia de Alimentos (DEA), Universidade do Estado de Santa Catarina (UDESC), Pinhalzinho, SC.

** Doutor em Física, professor do DEA, UDESC, Pinhalzinho, SC.

*** Doutor em Engenharia Química, professor do DEA, UDESC, Pinhalzinho, SC. (e-mail: alessandro.galvao@ udesc.br) 


\section{INTRODUÇÃO}

A rápida degradação dos produtos de origem animal e a exigência de serem mantidas determinadas características do material desde o abate até o consumidor final ressaltam a necessidade de conservar, ao longo de toda a cadeia do frio, padrões estabelecidos de temperatura.

A cadeia do frio compreende todo o processo de armazenamento, conservação, distribuição, transporte e manipulação dos produtos, com o controle da baixa temperatura. Qualquer falha nessa cadeia pode comprometer a qualidade dos produtos, pois as velocidades das reações químicas, bioquímicas e microbiológicas são relacionadas diretamente com a temperatura, influenciando a sanidade e a qualidade nutricional e sensorial dos produtos refrigerados (PEREIRA et al., 2010).

O método mais empregado na indústria de cárneos para congelamento consiste na utilização de túneis ou salas equipadas com ventiladores nos quais um fluxo de ar forçado constitui o meio de transferência de calor. A energia consumida pelo equipamento durante o processo de congelamento é de suma importância para o processo industrial, sendo esse fator intimamente ligado a uma eficiente distribuição espacial da velocidade do ar ao redor do produto que será congelado (RESENDE et al., 2002).

Dentre os fatores que influenciam a taxa de resfriamento por ar forçado, além da diferença de temperatura entre o fluxo de ar e o material a ser congelado, estão a área de superfície de contato, a velocidade do ar de resfriamento e a orientação da montagem e empilhamento do produto a ser resfriado no interior do túnel de congelamento (NEVES FILHO et al., 2009).

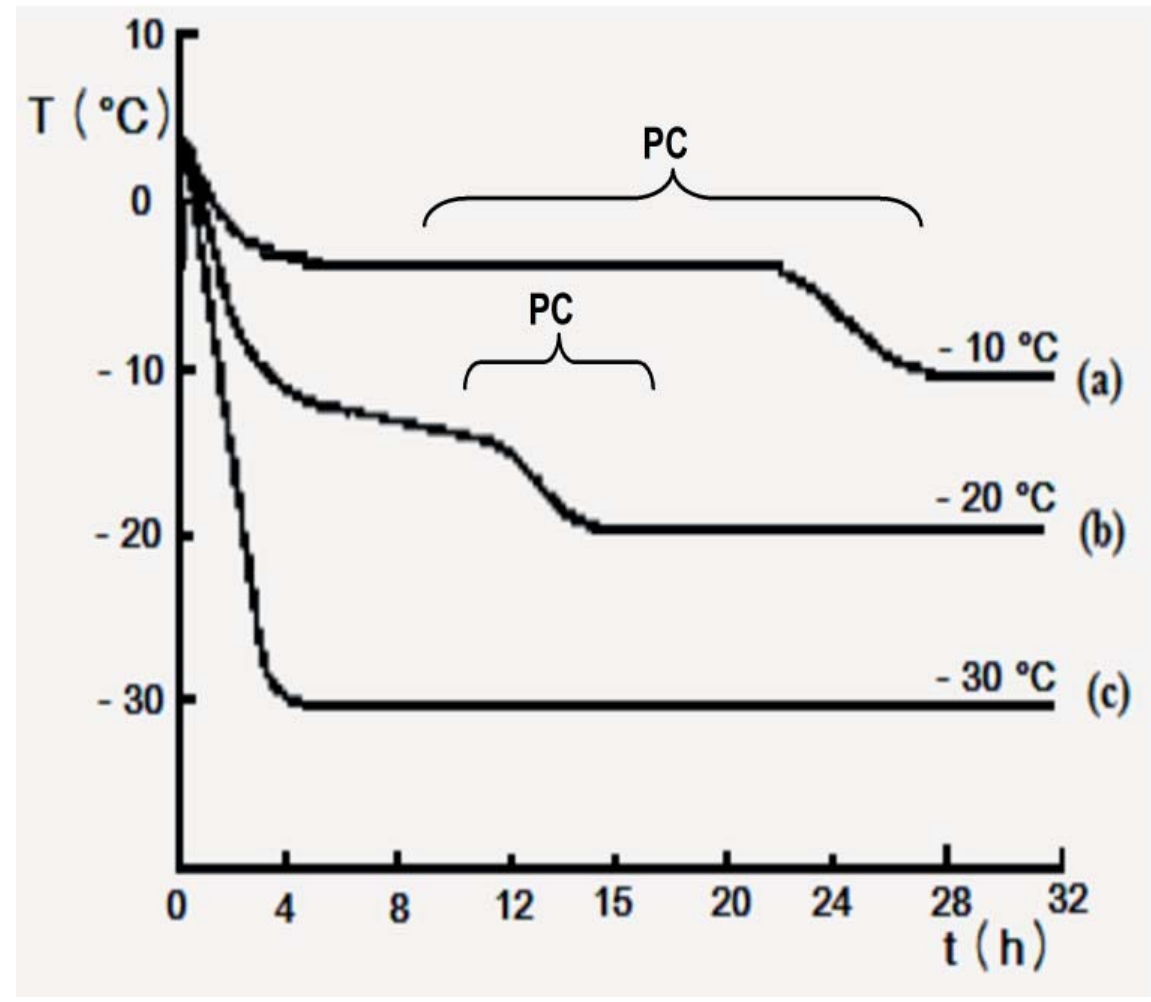

FIGURA 1 - CURVAS DE CONGELAMENTO MOSTRANDO AS VELOCIDADES RELATIVAS DE CONGELAMENTO A DIFERENTES TEMPERATURAS

(FORREST ET AL., 1979). 
Outra questão importante é a distribuição do ar em relação à geometria da câmara e a uniformidade da trajetória do escoamento. Nesse contexto, o controle do escoamento de ar é um ponto crítico na instalação, pois o perfil de velocidade do escoamento determina a eficiência e a homogeneidade dos tratamentos aos quais o produto está sendo submetido (RESENDE et al., 2002).

Na operação de congelamento, a diminuição da temperatura no centro térmico do material pode ser conduzida de forma lenta ou rápida. Durante o congelamento lento, a temperatura do produto permanece próxima ao ponto de congelamento inicial por um longo tempo, apresentando um período de cristalização (PC). No congelamento rápido, a temperatura do produto cárneo a ser congelado cai rapidamente abaixo do ponto de congelamento inicial, não apresentando período de cristalização. A figura 1 ilustra o comportamento da temperatura de um produto durante 0 congelamento. As curvas "a" e "b" representam o congelamento lento com PC, e a curva "c", o congelamento rápido, não apresentando PC.

O período de cristalização consiste na formação de cristais de gelo extracelulares grandes que causam a separação física das fibras, formando ranhuras alternadas por fibras e cristais de gelo. Um produto cárneo que foi submetido a um congelamento lento, durante o descongelamento, apresenta um "gotejamento", ou seja, a perda de fluido intracelular de células que foram rompidas durante o congelamento. No congelamento rápido, os cristais formados são intracelulares e pequenos, de forma que, no descongelamento, os cristais são facilmente reabsorvidos pelas células, não apresentando, dessa forma, perda de material intracelular, que leva a uma diminuição da qualidade do produto cárneo.

O produto cárneo deve permanecer no túnel de congelamento o tempo necessário para que a temperatura no seu centro térmico seja reduzida ao padrão desejado (SANTOS, 2005), podendo então ser transferido para uma câmara de estocagem para a manutenção dessa condição até o momento da comercialização. No Brasil, o Ministério da Agricultura, Pecuária e Abastecimento (BRASIL, 1995) estabelece que os túneis de congelamento rápido devem operar a uma temperatura entre $-35^{\circ} \mathrm{C}$ e $-40^{\circ} \mathrm{C}$, com velocidade do ar entre $5 \mathrm{~m} / \mathrm{s}$ e $6 \mathrm{~m} / \mathrm{s}$.

Diante do apresentado, o presente trabalho teve por objetivo avaliar as características do congelamento do carré suíno realizado por uma empresa localizada na região oeste do estado de Santa Catarina, Brasil. Na condução do trabalho, foram realizadas análises do perfil da velocidade de congelamento em relação à velocidade do ar em diferentes pontos do túnel de congelamento.

\section{MATERIAL E MÉTODOS}

Foram selecionados três pontos no túnel de congelamento, de modo a proporcionar uma leitura de temperaturas e velocidade do ar ao longo da seção perpendicular ao fluxo de ar. A figura 2 ilustra a posição dos pontos de estudo (P1, P2 e P3) no túnel de congelamento.

Para a realização dos experimentos foram selecionados cortes de carré suíno realizados pela empresa. Os cortes foram acondicionados em caixas de papelão padrão da empresa, com as tampas abertas durante o período de congelamento. As caixas foram acondicionadas em gaiolas metálicas e colocadas nos diferentes pontos selecionados para o estudo.

Foi arbitrado um intervalo de tempo de 30 minutos para a leitura das temperaturas do núcleo do carré suíno $\left(T_{N}\right)$, da superfície do produto $\left(T_{S}\right)$ e do túnel de congelamento $\left(T_{T}\right)$. Na determinação da $T_{N}$ e da $T_{S}$ foi utilizado um sensor PT100, parte integrante de um DataLogger da marca LogBox, modelo LogChart II, versão 2.34. E, na determinação da $T_{T}$, foi utilizado um sensor PT100 instalado no centro do túnel, localizado a aproximadamente um metro de distância do evaporador. 


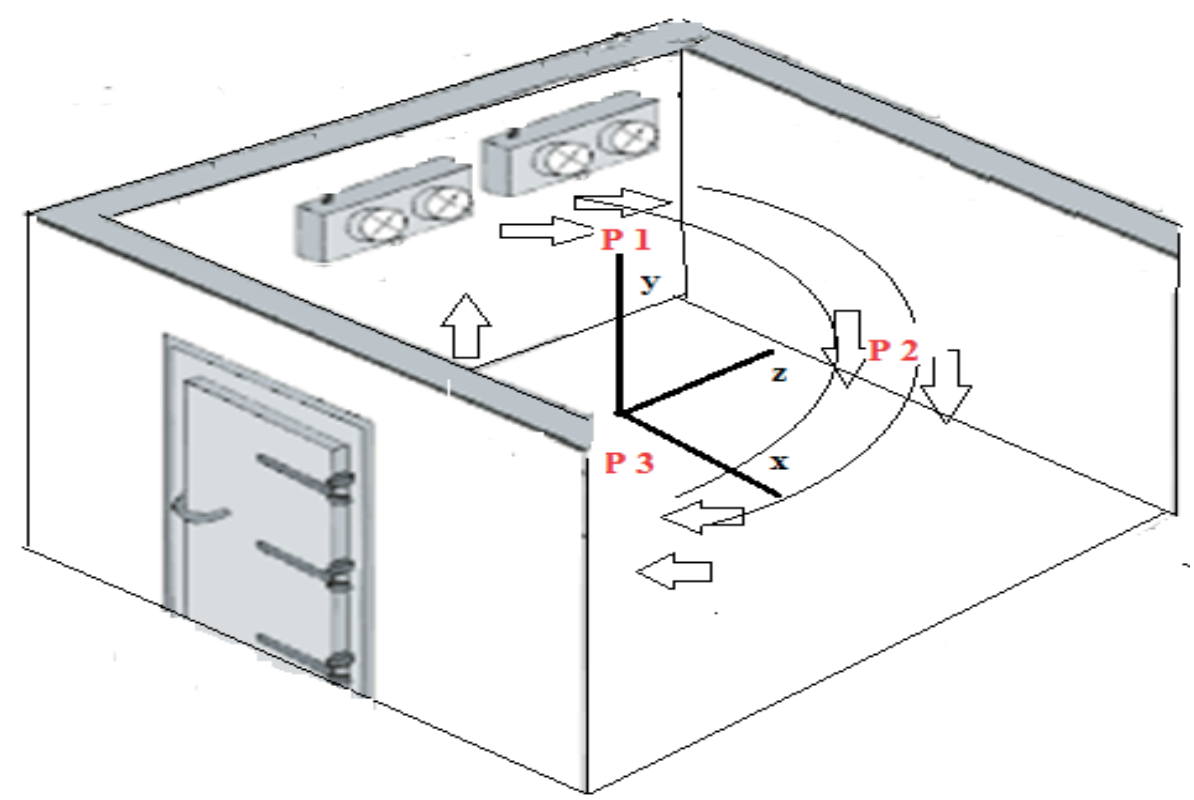

\section{FIGURA 2 - PONTOS SELECIONADOS PARA O CONGELAMENTO DO CARRÉ SUÍNO NO TÚNEL DE CONGELAMENTO.}

A determinação do tempo de congelamento foi realizada tendo como referência a temperatura no núcleo do carré suíno, de $-12{ }^{\circ} \mathrm{C}$. Essa temperatura, além de ser o valor adotado pela empresa na expedição do produto para o mercado interno, também é comumente utilizada no mercado brasileiro por representar um parâmetro em que as atividades enzimática e microbiológica são muito baixas no alimento.

As leituras da velocidade do ar no túnel foram feitas com um anemômetro da marca Minipa, modelo MDA 11, nos pontos selecionados para o estudo, registrando-se os valores máximos e mínimos de velocidade do ar em um intervalo de leitura de 16 segundos, conforme programação do equipamento. O sensor do anemômetro foi inserido entre as caixas de carré suíno na área perpendicular ao fluxo de escoamento do ar nos diferentes pontos selecionados para o estudo. Com os dados de velocidade do ar, utilizou-se a média dos valores encontrados em cada ponto do túnel.

Com a finalidade de avaliar uma condição crítica da operação, o monitoramento do congelamento do carré suíno nos diferentes pontos do túnel foi realizado nos dias em que o túnel se encontrava com capacidade máxima.

\section{RESULTADOS E DISCUSSÕES}

A figura 3 apresenta o perfil de congelamento do carré suíno para as três regiões do túnel de congelamento avaliadas em relação às temperaturas da superfície do produto e do túnel.

Os resultados apresentam constantes variações na temperatura do túnel devido à frequentes aberturas de porta realizadas durante a operação de congelamento. Cada abertura de porta da câmara proporciona a troca do ar refrigerado pelo ar quente e úmido do ambiente externo. Quando as aberturas de porta são frequentes, existe a indesejável formação de gelo no evaporador do sistema de refrigeração, reduzindo o desempenho do refrigerador e aumentando a necessidade da realização de degelos, especialmente em ambientes com alta umidade relativa. O gelo formado dificulta a troca de calor entre a superfície da serpentina e o ar circulado pela câmara e pode, inclusive, bloquear totalmente a sua passagem pela serpentina. 

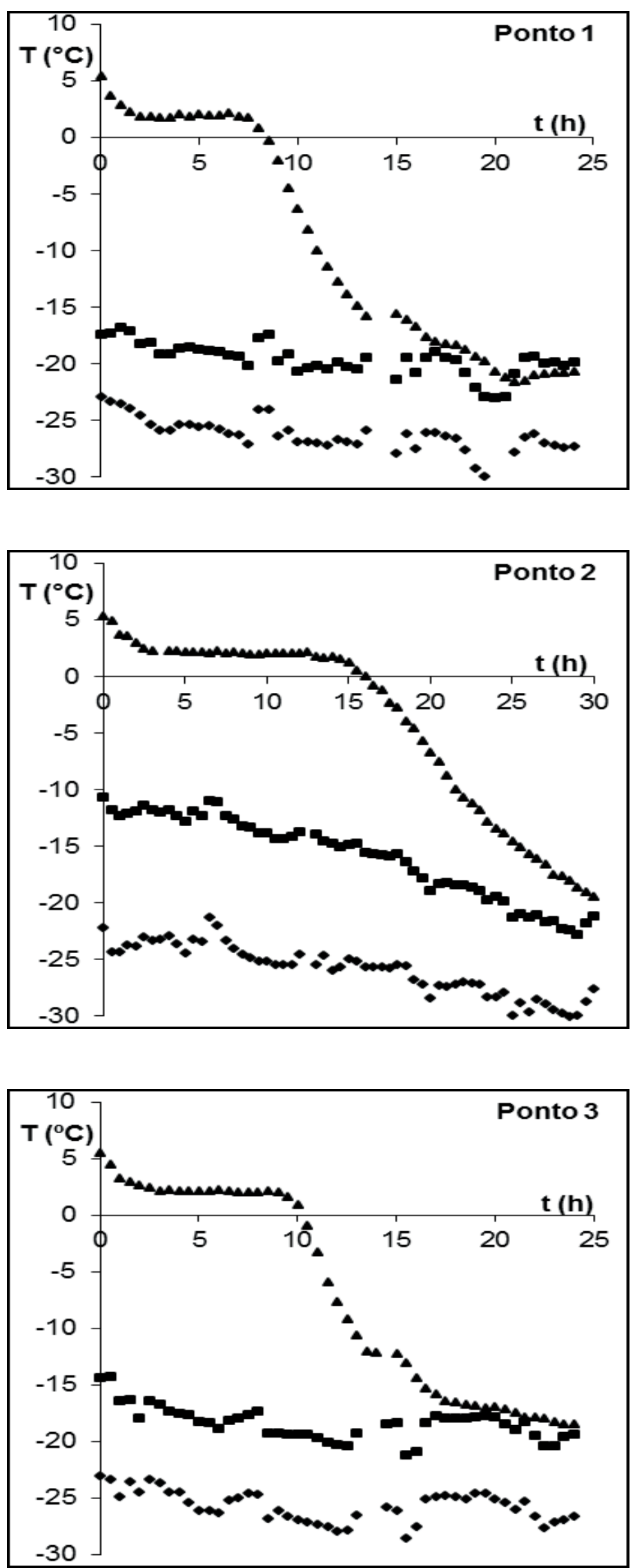

FIGURA 3 - CURVAS DE CONGELAMENTO DO CARRÉ SUÍNO NAS REGIÕES DA

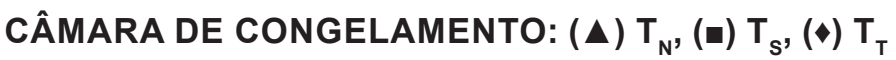


Calculando-se a diferença entre a temperatura do túnel e a temperatura da superfície do produto (ÄT), pode-se observar com maior nitidez o comportamento do congelamento em cada ponto estudado, conforme apresentado na figura 4 . O ponto 2 apresenta as maiores variações de temperatura, com valores decrescentes ao longo do período de congelamento. Os pontos 1 e 3 possuem uma variação de temperatura semelhante, com comportamento aproximadamente constante ao longo do congelamento. As variações de temperatura existentes entre cada ponto avaliado e o túnel de congelamento podem ser justificadas pelas constantes aberturas de porta durante a operação do equipamento, de forma que, dos três pontos estudados, o ponto 2 é a região do túnel que é mais afetada por esse comportamento.

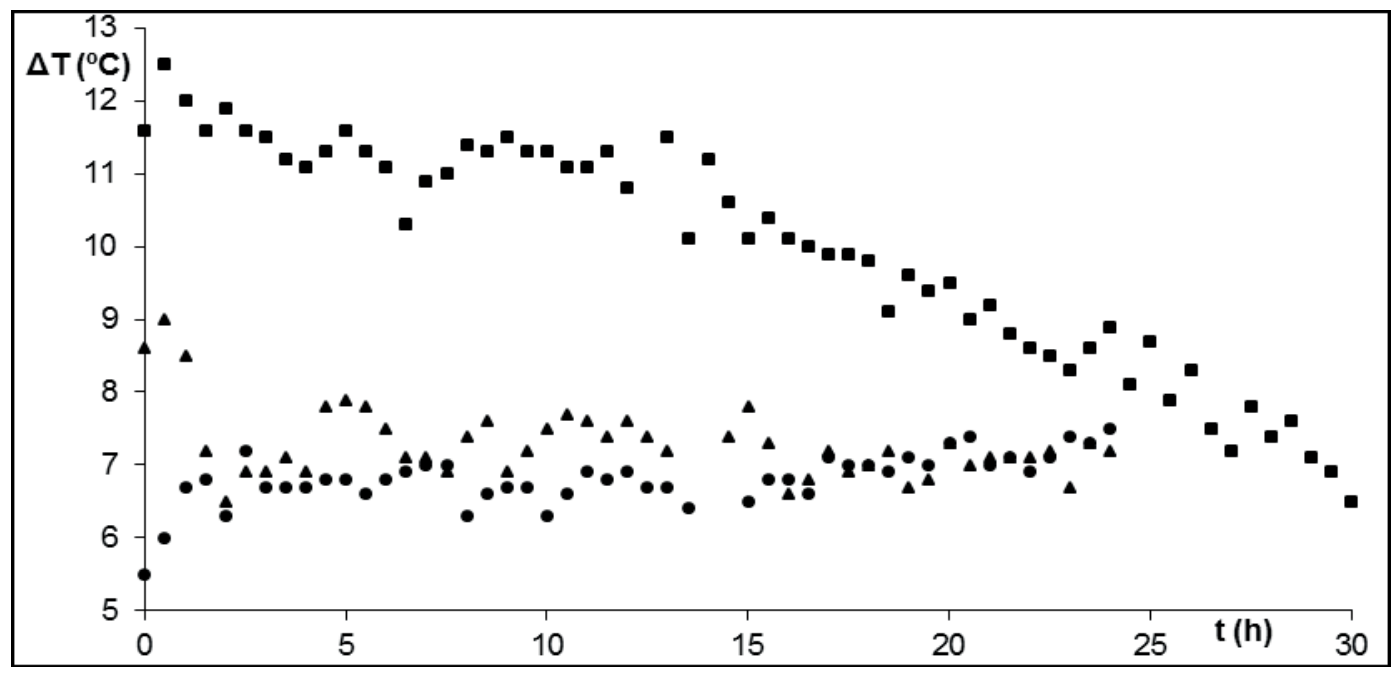

FIGURA 4 - VARIAÇÃO DE TEMPERATURA (ÄT) ENTRE O TÚNEL E A SUPERFÍCIE DO PRODUTO: (•) PONTO 1, (घ) PONTO 2, ( 1 ) PONTO 3

A temperatura de operação do túnel de congelamento representa fator determinante no tempo e na qualidade do congelamento. Observa-se que o túnel avaliado em nenhum momento operou com temperatura inferior a $-30^{\circ} \mathrm{C}$, de forma que esse comportamento pode ter contribuído negativamente no resfriamento, visto que os três perfis de congelamento, conforme apresentado pela figura 5 , apresentam período de cristalização, característica inerente ao congelamento lento.

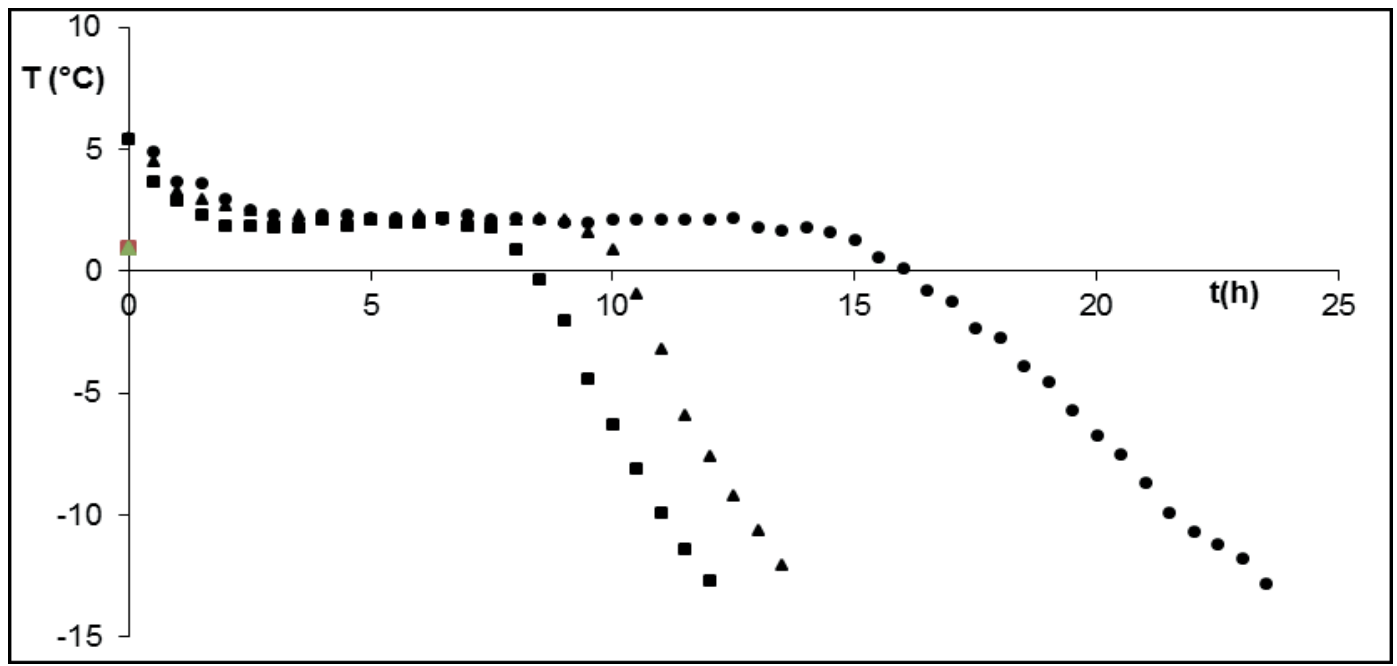

FIGURA 5 - TEMPERATURA DO NÚCLEO PARA O CONGELAMENTO DE CARRÉ SUÍNO: (ロ) PONTO 1, (•) PONTO 2, ( ) PONTO 3 
Observa-se que o período de cristalização no ponto 2 é duas vezes maior que o período de cristalização apresentado no ponto 1. Quando os pontos 1 e 3 são comparados, percebe-se que o período de cristalização no ponto 3 é superior ao apresentado pelo ponto 1 . A existência de período de cristalização afeta diretamente o tempo de congelamento, sendo que o ponto 2 apresenta o pior tempo de congelamento, consumindo um período superior a 23 horas. Os pontos 1 e 3 apresentaram resultados bem inferiores, com 12 horas e 13,5 horas de operação respectivamente.

Após o período de cristalização, observa-se um comportamento linear da temperatura de resfriamento que permite determinar a velocidade de congelamento expressa pelo coeficiente angular da reta que correlaciona os pontos experimentais, conforme representado na figura 6 .



FIGURA 6 - CORRELAÇÃO LINEAR DA TEMPERATURA DO NÚCLEO DO CARRÉ SUÍNO: (ロ) PONTO 1, (•) PONTO 2, ( 4 ) PONTO 3

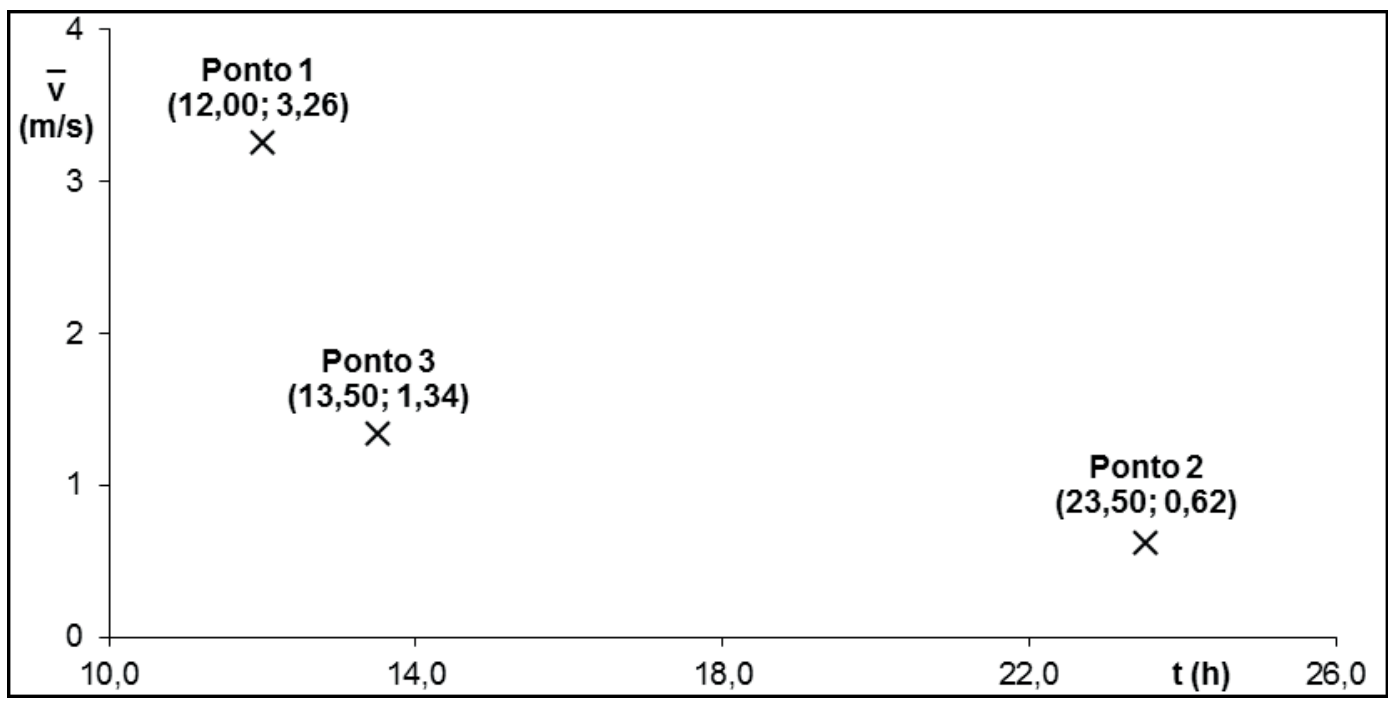

FIGURA 7 - RELAÇÃO ENTRE O TEMPO DE CONGELAMENTO E A VELOCIDADE MÉDIA DO FLUXO DE AR 
Analisando-se as velocidades de congelamento, verifica-se que os pontos 1 e 3 do túnel de congelamento possuem os melhores resultados de congelamento após o período de cristalização, observando-se um comportamento de congelamento bastante similar, com decaimento de temperatura de aproximadamente $3,4{ }^{\circ} \mathrm{C}$ por hora de operação do túnel. Em contrapartida, o ponto 2 expressa o pior comportamento, apresentando um decaimento de temperatura de aproximadamente $1,7^{\circ} \mathrm{C}$ por hora de operação do túnel. A avaliação dos resultados indica que o ponto 2 necessita do dobro do tempo de operação para alcançar a temperatura desejada quando comparado aos demais pontos em estudo.

Em relação à velocidade média do fluxo de ar, verifica-se que cada ponto do túnel é refrigerado de forma distinta. O ponto 1 apresentou velocidade média de $3,26 \mathrm{~m} / \mathrm{s}$; o ponto 2 , de $0,62 \mathrm{~m} / \mathrm{s}$; e o ponto 3 , de $1,37 \mathrm{~m} / \mathrm{s}$. Uma relação entre o tempo de congelamento e a velocidade média do ar é apresentada na figura 7. Observa-se que a velocidade do ar no interior do túnel de congelamento representa uma variável fundamental no resfriamento do carré suíno, havendo influência direta no tempo de congelamento do produto, de modo que maiores velocidades levam a menores tempos de congelamento. O comportamento inconstante e não linear entre velocidade média do fluxo de ar e tempo de congelamento pode ser atribuído às mudanças bruscas no direcionamento do escoamento do ar, devido à formação de caminhos preferenciais relacionados à presença de obstáculos inerentes ao volume e à distribuição do material no interior do túnel de congelamento.

\section{CONCLUSÃO}

O tempo de congelamento no sistema de refrigeração por ar forçado é função da temperatura do meio, da velocidade do fluxo de ar, da disposição e geometria dos produtos e de sua posição dentro do túnel de congelamento.

Durante o estudo, o túnel avaliado apresentou constantes variações na sua temperatura, consequência das aberturas de porta durante o congelamento dos cortes. As portas dos túneis devem ficar abertas somente pelo tempo necessário, devendo ser fechadas o mais rápido possível.

As curvas de congelamento analisadas indicam comportamento de congelamento lento. A existência de período de cristalização nos três pontos investigados indica uma operação de congelamento não satisfatória. O período de cristalização no congelamento de cárneos deve ser o menor possível, pois o número e o tamanho dos cristais de gelo afetam diretamente a qualidade sensorial dos produtos.

Períodos de cristalização levam a um aumento desnecessário no tempo de congelamento, proporcionando uma repercussão negativa no consumo de energia e na capacidade do equipamento.

Os diferentes resultados de velocidade média do ar e tempo de congelamento indicam que o túnel de congelamento não operou de forma eficiente no período avaliado. A distribuição ruim do ar frio no interior do túnel pode ter sido causada pela existência de caminhos preferenciais do fluxo de ar ou pela disposição do material a ser congelado em relação à geometria do túnel.

O processo de congelamento analisado pode ser melhorado por meio do investimento em túneis de congelamento contínuos, compressores mais eficientes e uso de defletores para melhor distribuição do ar refrigerado. Além da melhora na qualidade do congelamento, sistemas mais eficientes consomem menor quantidade de energia, proporcionando uma economia financeira que em um certo período de tempo compensa o investimento realizado. 


\section{FREEZING PROFILE OF PORK LOIN: A CASE STUDY}

\section{ABSTRACT}

This study had the purpose of verifying the features of a freezing tunnel owned by a Brazilian company located at the western part of Brazil. It was performed velocity profile analysis of the pork loin freezing as a function of the air velocity in different locations of the tunnel. A detailed analysis of the data showed the existence of two different periods of freezing. This behavior indicates that the freezing of pork loin in the investigated tunnel is not proper. The analysis of the average speed of air for all the three areas of the tunnel point to a large heterogeneity in the freezing operation, leading to a direct effect in meat quality and in the time of freezing.

KEY-WORDS: CASE STUDY, FREEZING, PORK LOIN.

\section{REFERÊNCIAS}

1 BALLIN, N.Z.; LAMETSCH, R. Analytical methods for authentication of fresh vs. thawed meat: A review. Meat Science, v. 80 , n. 2, p. 151-158, oct. 2008.

2 BRASIL. Ministério da Agricultura, Pecuária e Abastecimento. Portaria n. 711 de 01 de novembro de 1995. Normas técnicas de instalações e equipamentos para abate e industrialização de suínos. Diário Oficial da União, Brasília, 03 de novembro de 1995.

3 FORREST, J.C.; ABERLE, E.D.; HEDRICK, H.B.; JUDGE, M.D.; MERKEL, R.A. Fundamentos de ciencia de la carne. Zaragoza: Acribia, 1979. 363 p.

4 KIANI, H.; SUN, D.-W. Water crystallization and its importance to freezing of foods: A review. Trends in Food Science \& Technology, v. 22, p. 407-426, aug. 2011.

5 KIM, Y.H.B.; LIESSE, C.; KEMP, R.; BALAN, P. Evaluation of combined effects of ageing period and freezing rate on quality attributes of beef loins. Meat Science, v. 110, p. 40-45, dec. 2015.

6 LEYGONIE, C.; BRITZ, T.J.; HOFFMAN, L.C. Impact of freezing and thawing on the quality of meat: Review. Meat Science, v. 91, p. 93-98, jun. 2012.

7 MUELA, E.; SANUDO, C.; CAMPO, M.M.; MEDEL, I.; BELTRAN, J.A. Effect of freezing method and frozen storage duration on instrumental quality of lamb throughout display. Meat Science, v. 84, p. 662-669, apr. 2010.

8 MUELA, E.; SANUDO, C.; CAMPO, M.M.; MEDEL, I.; BELTRAN, J.A. Effect of freezing method and frozen storage duration on lamb sensory quality. Meat Science, v. 90, p. 209-215, jan. 2012.

9 NEVES FILHO, L.C.; BARBIN, D.F.; SILVEIRA JUNIOR, V. Processo de congelamento em túnel portátil com convecção forçada por exaustão e insuflação para paletes. Ciência e Tecnologia de Alimentos, v. 29, n. 3, p. 667-675, jul.-set. 2009.

10 PEREIRA, V.F.; DORIA, E.C.B.; CARVALHO JÚNIOR, B.C.; NEVES FILHO, L.C.; SILVEIRA JUNIOR, V. Avaliação de temperaturas em câmaras frigoríficas de transporte urbano de alimentos resfriados e congelados. Ciência e Tecnologia de Alimentos, v. 30, n. 1, p. 158-165, jan.-mar. 2010.

11 PIETRASIK, Z;; JANZ, J.A.M. Influence of freezing and thawing on the hydration characteristics, quality, and consumer acceptance of whole muscle beef injected with solutions of salt and phosphate. Meat Science, v. 81, n. 3, p. 523-532, mar. 2008.

12 RESENDE, J.V.; NEVES FILHO, L.C.; SILVEIRA JUNIOR, V. Escoamento de ar através de embalagens de polpa de frutas em caixas comerciais: efeitos sobre os perfis de velocidade em túneis de congelamento. Ciência e Tecnologia de Alimentos, v. 22, n. 2, p. 184-191, maio-ago. 2002.

13 SANTOS, C.A. Estudo da Transferência de Calor Durante o Congelamento de Cortes de Aves em Caixas de Papelão Ondulado. 2005. 99 p. Dissertação (Mestrado em Engenharia de Alimentos) - Programa de Pós-Graduação em Engenharia de Alimentos, Universidade Federal de Santa Catarina, Florianópolis, 2005.

14 SHINBAYEVA, A.; ALDIYAROV, A.; ARKHAROV, I.; DROBYSHEV, A.S. Investigations of the dynamics of meat freezing at various modes of cryotreatment. Physical Sciences and Technology, v. 1, n. 2, p. 36-40, 2014. 
